Thirdly it follows easily from these observations and the residual nilpotence of free groups that $G$ is parafree of rank $r$.

Finally we observe that $G$ is not finitely generated, but that $G / \gamma_{2} G$ is free abelian of rank two. Hence $G$ is not free.

\title{
REFERENCES
}

1. G. Baumslag, Groups with the same lower central sequence as a relatively free group. I: The groups, Trans. Amer. Math. Soc. 129 (1967), 308-321.

2. - Some groups that are just about free, Bull. Amer. Math. Soc. 73 (1967), 621-622.

Graduate Center, The City University of New York

\section{SOLVABLE AND NILPOTENT SUBALGEBRAS OF LIE ALGEBRAS}

\author{
BY DAVID J. WINTER ${ }^{1}$ \\ Communicated by G. B. Seligman, February 21, 1968
}

1. Introduction. We describe here some results on the structure of a Lie algebra in terms of its nilpotent and solvable subalgebras. Proofs will appear elsewhere.

In the following discussions, $F$ is an arbitrary field, $\mathscr{L}$ is a (finite dimensional) Lie algebra over $F$ and $V$ is a (finite dimensional) vector space over $F$.

2. Arbitrary Lie algebras. Let $\Re$ be a set of linear transformations in $V$ such that the Lie algebra generated by $\Re$ over $F$ is nilpotent. Then, as is well known, $V$ has a unique vector space decomposition $V=V_{0}(\Re)+V_{*}(\Re)$ (direct) where $V_{*}(\Re)$ is $\Re$-stable, $V_{0}(\Re)$ is $\Re$-stable and $\left.\Re\right|_{V_{0}(\Re)}$ consists of nilpotent transformations, and where $V_{0}(\Im)$ is maximal with respect to the latter two properties.

One has the following theorem, in spite of the fact that a nilpotent linear Lie algebra cannot always be triangulized over the algebraic closure of its base field.

TheOREM $1 .{ }^{2}$ If $F$ is infinite and $\Re$ is a subspace of $\operatorname{Hom}_{F}(V, V)$ such that the Lie algebra generated by $\mathfrak{N}$ is nilpotent, then there exists $N$ in $\Re$ such that $V_{0}(N)=V_{0}(\Re)$.

1 This research was done while the author was a National Science Foundation Postdoctoral Research Fellow at the University of Bonn.

${ }^{2}$ This is essentially Lemma 3.5 of [4, pp. 87-88]. The author has heard that an independent forthcoming paper of $\mathrm{R}$. Block contains material close to this theorem. 
Definition. Cart $\mathscr{L}$ is the set of Cartan subalgebras of $\mathscr{L}$.

DEFINITION. Let $0 \rightarrow \mathscr{L}^{\prime} \stackrel{\alpha}{\rightarrow} \stackrel{\beta}{\rightarrow} \overline{\mathscr{L}} \rightarrow 0$ be an exact sequence of Lie algebras over the field $F$. Then $\alpha^{*}$ is the function with domain eart $\mathscr{L}$ defined by $\alpha^{*}(\mathfrak{H C})=\mathfrak{L}_{0}^{\prime}\left(\operatorname{ad}\left(\mathcal{H C} \cap \mathfrak{L}^{\prime}\right)\right)$ and $\beta_{*}$ the function with domain eart $\mathscr{L}$ defined by $\beta_{*}(\mathfrak{H C})=\beta(\mathfrak{H C})$ for $\mathcal{T C}$ in Cart $\mathcal{L}$. (Here and later, $\alpha$ is assumed to be inclusion.)

Basic properties of $\beta_{*}$ have been independently noticed by $D . W$. Barnes [6], R. Block, and the author. In particular, $\beta_{*}$ maps Cart $\mathscr{L}$ into Cart $\overline{\mathscr{L}}$.

In the next section, properties of the function $\alpha^{*}$ and its relations to $\beta_{*}$ are described.

\section{Lie algebras of characteristic 0 and restricted Lie algebras.}

THEOREM 2. Let $0 \rightarrow \mathfrak{L}^{\prime} \stackrel{\alpha}{\rightarrow} \mathfrak{L} \stackrel{\beta}{\rightarrow} \overline{\mathscr{L}} \rightarrow 0$ be an exact sequence of Lie algebras. Suppose that characteristic $F$ is 0 or that characteristic $F$ is $p>0$ and $\left(\operatorname{ad}_{\mathfrak{L}^{\prime}} \mathfrak{L}^{\prime}\right)^{p} C \operatorname{ad}_{\mathfrak{L}^{\prime}} \mathcal{L}^{\prime},\left(\operatorname{ad}_{\mathfrak{L}} \mathfrak{L}\right)^{p} \mathrm{C} \operatorname{ad}_{\mathfrak{L}} \mathcal{L}$. Then the function $\alpha^{*}$ with domain Cart $\mathcal{L}$ defined in the above section has the following properties:

(1) Image $\alpha^{*}=$ Cart $\mathfrak{L}^{\prime}$;

(2) for $\mathfrak{H C}^{\prime}$ in Cart $\mathfrak{L}^{\prime}, \alpha^{*-1}\left(\mathcal{H C}^{\prime}\right)=$ Cart $\mathfrak{L}_{0}\left(\operatorname{ad} \mathfrak{H C}^{\prime}\right)$.

The properties (1) and (2) are conveniently represented by the following "exact sequences," where $\mathfrak{H C}^{\prime}$ ranges over Cart $\mathfrak{L}^{\prime}$ :

$$
0 \leftarrow \text { eart } \mathfrak{L}^{\prime} \stackrel{\alpha^{*}}{\leftarrow} \text { Cart } \mathfrak{L} \leftarrow \text { Cart } \mathfrak{L}_{0}\left(\operatorname{ad} \mathfrak{H}^{\prime}\right) \leftarrow 0 \text {. }
$$

THEOREM 3. Let the hypothesis be as in Theorem 2. Then for any $\mathrm{JC}^{\prime}$ in Cart $\mathfrak{L}^{\prime}$ and any $\mathcal{H}$ in Cart $\mathcal{L}$, the following conditions are equivalent:

(1) $\mathfrak{H C}^{\prime}=\alpha^{*}(\mathfrak{H C})$;

(2) $\mathfrak{H}$ normalizes $\mathfrak{H C}^{\prime}$;

(3) $\mathfrak{H C} \cap \mathbb{L}^{\prime} \subset \mathfrak{H C}^{\prime}$.

THEOREM 4. Let the hypothesis be as in Theorem 2. For JC in Cart \&, let $\mathfrak{H C}^{\prime}=\alpha^{*}(\mathfrak{H C})$ and $\overline{\mathfrak{H}}=\beta_{*}(\mathfrak{H C})$. Then $\mathfrak{H C}$ normalizes $\mathfrak{H C}^{\prime}$ and the subsets Cart $\mathfrak{L}_{0}\left(\operatorname{ad} \mathfrak{H C}^{\prime}\right)$, eart $\beta^{-1}(\overline{\mathcal{H C}})$ of eart $\mathfrak{L}$ satisfy the following properties:

(1) Cart $\mathfrak{L}_{0}\left(\operatorname{ad} \mathfrak{H C}^{\prime}\right) \cap \mathcal{C a r t} \beta^{-1}(\overline{\mathfrak{F C}})=$ Cart $\mathfrak{H C}+\mathfrak{H C}^{\prime}$;

(2) if $F$ is infinite, eart $\mathfrak{L}_{0}\left(\operatorname{ad} \mathfrak{H}^{\prime}\right)=\operatorname{Cart} \beta^{-1}(\mathfrak{H C})$ if and only if $\mathfrak{L}^{\prime}$ is nilpotent and $\mathfrak{L}^{\infty} \subset \mathfrak{L}^{\prime}$.

It is known that there are solvable Lie algebras over algebraically closed fields of characteristic $p>0$ having Cartan subalgebras of different dimensions. This provides a strongly negative answer to the question of conjugacy of Cartan subalgebras of an arbitrary Lie algebra. The situation is, however, for the present important class of 
Lie algebras not too bad, as is indicated by the remaining material of this section.

Definition. Let $\mathfrak{T}$ be a linear Lie algebra over $F$. A torus of $\Re($ is a subalgebra of $\Re($ which, upon extension of the base field of $\Re(t$ to its algebraic closure, becomes diagonalizable. A semisimple element of $\mathfrak{N}$ is an element of $\mathfrak{T}$ which is contained in some torus of $\mathfrak{T}$. A torus of $\mathscr{T C}$ is $F$-split if it is diagonalizable over $F$ itself. If $\Re^{p} \subset \mathscr{C}, \mathfrak{T}$ is said to be $F$-split if $\mathfrak{T C}$ has a torus of maximal dimension which is $F$-split.

Definition. A subalgebra $\mathfrak{H C}$ of $\mathcal{L}$ is of maximal ad-rank if ad $\mathfrak{H C}$ contains a torus of ad $\&$ of maximal dimension.

The next theorem insures the existance of a large number of Cartan subalgebras of maximal ad-rank.

Theorem 5. If characteristic $F$ is $p>0$, and if $(\mathrm{ad} \&)^{p} \mathrm{Cad} \&$ and ad $\mathcal{L}$ is $F$-split, then $\mathcal{L}$ is spanned as vector over $F$ by the union of all Cartan subalgebras of maximal ad-rank.

Definition. $\operatorname{Cart}_{\operatorname{Max}} \mathcal{L}=\{\mathcal{F} \in \mathcal{C}$ art $\& \mid \mathcal{F}$ is of maximal ad-rank $\}$.

Definition. For $x$ in $\mathscr{L}$, eart $\mathbb{L}=\left\{\mathcal{L} \in \operatorname{Cart}_{\mathrm{Max}} \mathscr{L} \mid x \in \mathcal{H C}\right.$ and $x$ is a common eigenvector for all of the semisimple elements of $\left.\operatorname{ad}_{\mathfrak{L}} \mathfrak{H}\right\}$.

For $\mathcal{H C}$ in $\operatorname{Cart}_{x} \mathscr{L}$, let $\mathfrak{F C}_{s}(1+x)=\{h(I+\operatorname{ad} x) \mid$ ad $h$ is a semisimple element of $\left.\operatorname{ad}_{\mathscr{L}} \mathfrak{F C}\right\}$. The Lie algebra generated by ad $\mathfrak{F C}_{s}(1+x)$ is nilpotent, so the following definition makes sense.

Definition. $e^{x}$ is the function with domain eart $_{x} \&$ defined for $\mathfrak{F}$ in $\operatorname{Cart}_{x} \&$ by $\mathcal{H C} e^{x}=\mathscr{L}_{0}\left(\operatorname{ad} \mathfrak{F C}_{8}(1+x)\right)$.

TheOREM 6. If characteristic $F$ is $p>0$ and $(\operatorname{ad} \mathscr{L})^{p} C \operatorname{ad} \mathscr{L}$, then $e^{x}$ is a bijection from Cart $_{x} \&$ onto itself with inverse $e^{-x}$.

In the case that characteristic $F$ is 0 and ad $\&$ is almost algebraic, $e^{x}$ is the operation on eart $_{x} \mathscr{L}$ determined by the automorphism exp ad $x=\sum_{0}^{\infty}(1 / n !)(\operatorname{ad} x)^{n}$ of $\mathscr{L}$. (In the present case, exp ad $x$ exists unless ad $x$ is not nilpotent, and if ad $x$ is not nilpotent, $\operatorname{eart}_{x} \mathscr{L}$ is empty.)

THEOREM 7. Let $F$ be an algebraically closed field of characteristic $p>0$, and $\mathscr{L}$ a solvable Lie algebra such that $(\mathrm{ad} \mathscr{L})^{p} \mathrm{Cad} \mathscr{L}$. Then all Cartan subalgebras of $\mathfrak{L}$ are of maximal ad-rank. Moreover any two Cartan subalgebras $\mathfrak{H C}, \mathfrak{H C}^{\prime}$ of $\mathfrak{E}$ are conjugate in the sense that there exist Cartan subalgebras $\mathfrak{H C}_{1}, \cdots, \mathfrak{F}_{n}$ of $\mathfrak{L}$ and elements $x_{1}, \cdots, x_{n}$ of $L^{\infty}$ such that

(1) $\mathfrak{H C}_{i} \in \operatorname{Cart}_{x_{i}} \&$ for $1 \leqq i \leqq n$;

(2) $\mathfrak{H C}=\mathfrak{H C}_{1}, \mathfrak{F C}_{1} e^{x_{1}}=\mathfrak{H C}_{2}, \cdots, \mathfrak{H C}_{n} e^{x_{n}}=\mathfrak{F C}^{\prime}$. 
4. Linear Lie $p$-algebras. In this section, $\mathscr{L}$ denotes a linear Lie $p$-algebra, that is, a linear Lie algebra over a field $F$ of characteristic $p>0$ which is closed under $p$ th powers. It is well known that if $K$ is a field extension of $F$, then $\mathscr{L} \otimes_{F} K$ can be regarded canonically as a linear Lie $p$-algebra.

THEOREM 8. If $J$ is a maximal torus of $\mathbb{L}$ and $K$ an algebraic extension of $F$, then $\Im \otimes_{F} K$ is a maximal torus of $\mathfrak{L} \otimes_{F} K$.

THEOREM 9. If $\mathfrak{L}$ is solvable, the dimension of a maximal torus of $\mathfrak{L}$ is constant.

Definition. A nil subalgebra (nil ideal) of $\mathscr{L}$ is a subalgebra (ideal) of $\mathscr{L}$ consisting of nilpotent transformations. $\Re$ til $\mathscr{L}$ is a the sum of all nil ideals of $\mathscr{L}$. (It is easily seen that $\mathscr{T l i l} \mathscr{L}$ is a nil ideal of $\mathscr{L}$.)

TheOREM 10. Let $F$ be algebraically closed. Then if $\mathfrak{u}$ is a maximal nil subalgebra of $\mathfrak{L}$, the normalizer Torm $\mathfrak{U}$ of $\mathfrak{U}$ in $\mathfrak{L}$ is a maximal triangulizable subalgebra of $\&$.

The $\Re$ orm $\mathcal{U}$ of the above theorem is not always a maximal solvable subalgebra of $\mathcal{L}$. Also, it can happen, for $F$ algebraically closed, that $B$ is a maximal triangular subalgebra of $\&$ such that $\Re$ orm $B=B$, but $B$ is not maximal solvable in $\mathscr{L}$ and Til $B$ is not a maximal nil subalgebra of $\mathscr{L}$.

5. Algebraic Lie algebras. Throughout the remainder of the paper, we assume that $\mathscr{L}$ is the Lie algebra Lie $G$ of a connected linear algebraic group $G$ over an algebraically closed field $F$ having characteristic $p>3$. The adjoint representation of $G$ in Lie $G$ is denoted by Ad. The centralizer (respectively normalizer) in $\mathscr{L}$ of a subset $\mathfrak{T}$ of $\mathscr{L}$ is denoted by $\mathfrak{C}(\mathfrak{T})$ (respectively $\mathfrak{T}(\mathfrak{T})$ )). The centralizer (respectively normalizer) in $G$ of a subset $\mathscr{T C}$ of $\mathscr{E}$ or $G$ is denoted by $C(\mathfrak{T C}$ ) (respectively $N(\mathfrak{T})$ ). The connected component of the identity of a closed subgroup $H$ of $G$ is denoted by $H_{0}$.

Definition. A subalgebra $\mathbb{B}$ of $\mathfrak{L}$ is called a Borel subalgebra of $\mathbb{L}$ if $B$ is a maximal solvable subalgebra of $\&$ which contains a maximal torus of $\mathcal{L}$.

The following theorem shows that the above definition is equivalent to Definition 14.2 of [2], and is a step in the direction of an answer to an open question described in $\$ 14.3$. of [2].

THEOREM 11. $B$ is a Borel subalgebra of $\mathfrak{L}$ if and only if $N(B)$ is a Borel subgroup of $G$. The mapping with domain Bor $\mathscr{L}=\{\Theta \mid \Theta$ is Borel subalgebra of $\&\}$ defined by $B \rightarrow N(\mathbb{B})$ is a bijection from Bor $\mathbb{L}$ onto 
the set Bor $G$ of Borel subgroups of $G$. The inverse of this bijection is the mapping with domain Bor $G$ defined by $B \mid \rightarrow$ Lie $B$.

TheOREM 12. Let $\Im$ be a maximal torus of $\mathfrak{\&}=$ Lie G. Then:

(1) $C(\mathfrak{J})$ is connected and $N(\mathfrak{J})_{0}=C(\mathfrak{J})$;

(2) if $G$ is solvable, $N(\mathfrak{J})=C(\mathfrak{J})$;

(3) $W(\mathfrak{J})=N(\mathfrak{J}) / C(\mathfrak{J})$ permutes in a simply transitive manner the Borel subalgebras of $\&$ containing $\mathfrak{3}$. Moreover $W(\mathfrak{J})$ is canonically isomorphic to the Weyl group of $G$.

Though the groups $N(J)$ and $C(J)$ of the above theorem are often much larger than the groups $N(T), C(T)$ for $T$ a maximal torus of $G$, the quotients $N(J) / C(J), N(T) / C(T)$ are isomorphic.

\section{REFERENCES}

1. A. Borel and T. A. Springer, Rationality properties of linear algebraic groups, Proc. Sympos. Pure Math., Vol. 9, Amer. Math. Soc., Providence, R. I., 1966.

2. J. E. Humphreys, Algebraic groups and modular Lie algebras, Mem. Amer. Math. Soc., No. 71 (1967).

3. G. B. Seligman, Some results on Lie p-algebras, Bull. Amer. Math. Soc. 73 (1967), 528-530.

4. D. J. Winter, On automorphisms of Lie algebras and algebraic groups, Doctoral Dissertation, Yale University, New Haven, Conn., 1965.

5. - On groups of automorphisms of Lie algebras, J. Algebra 8 (1968), 131-142.

6. D. W. Barnes, On Cartan subalgebras of Lie algebras, Math. Z. 101 (1967), $350-355$.

University of Bonn, Germany 